\title{
Adherence to diabetic eye examination guidelines in Australia: the National Eye Health Survey
}

\author{
Joshua Foreman', Stuart Keel', Jing Xie ${ }^{1,2}$, Peter Van Wijngaarden', Hugh R Taylor ${ }^{3}$, Mohamed Dirani
}

The known Adherence to NHMRC diabetic eye examination guidelines in Australia is reported to be suboptimal, but the accuracy of estimates is questionable, as they are derived from studies with small sample sizes and recruitment bias.

The new In a national sample stratified by remoteness, about half of all Indigenous Australians with diabetes and almost one-quarter of non-Indigenous Australians with diabetes did not have their eyes examined at the recommended frequency, placing them at risk of vision-threatening retinopathy.

The implications Improving the provision and uptake of screening services is needed, particularly in Indigenous communities.

\section{$\mathrm{T}$} he number of adults with diabetes worldwide has almost quadrupled in the past 35 years, from 108 million in 1980 to 422 million in $2014 .^{1}$ This rise is largely attributable to population growth and ageing. ${ }^{2}$ In Australia, about 1.5 million adults have diabetes, and it is estimated that an additional 280 people are diagnosed each day. ${ }^{3}$ Diabetic retinopathy is a frequent complication of diabetes, and is the leading cause of blindness in working age adults in most developed nations. ${ }^{4}$ This is disturbing, as most blindness caused by diabetes can be avoided by early detection and timely intervention.

The National Health and Medical Research Council (NHMRC) has developed guidelines that recommend annual retinal screening and visual acuity assessment of Indigenous Australians and biennial assessment of non-Indigenous Australians with diabetes but without significant risk factors for retinopathy (longer diabetes duration, poor glycaemic control, elevated blood pressure or blood lipid levels). ${ }^{6}$ The difference in the recommended intervals for Indigenous and non-Indigenous Australians reflects epidemiological evidence that retinopathy is more common in Indigenous than in non-Indigenous Australians. ${ }^{7}$ Rates of adherence to these guidelines are reported to be unsatisfactory, as low as $50 \%$ for nonIndigenous Australians and $20 \%$ for Indigenous Australians, although the studies providing these estimates may have been limited by small sample sizes and recruitment bias.

Projections for the future prevalence of diabetes in Australian adults have been provided by the Australian Diabetes, Obesity and Lifestyle (AusDiab) study. ${ }^{9}$ If the incidence of diabetes observed during 2000-2005 continues, its prevalence will rise from $7.6 \%$ in 2000 to $11.4 \%$ by $2025 .^{9}$ As $25-35 \%$ of Australians with diabetes have some degree of retinopathy, it follows that a significant increase in the health impact and economic burden of diabetic retinopathy is likely.?

The National Eye Health Survey (NEHS) was undertaken to provide national prevalence data on vision impairment and blindness.

\section{Abstract}

Objective: To determine adherence to NHMRC eye examination guidelines for Indigenous and non-Indigenous Australian people with diabetes.

Design: Cross-sectional survey using multistage, random cluster sampling.

Setting: Thirty randomly selected geographic sites in the five mainland Australian states and the Northern Territory, stratified by remoteness.

Participants: 1738 Indigenous Australians aged 40-92 years and 3098 non-Indigenous Australians aged $50-98$ years were recruited and examined between March 2015 and April 2016 according to a standardised protocol that included a questionnaire (administered by an interviewer) and a series of standard eye tests.

Main outcome measures: Adherence rates to NHMRC eye examination guidelines; factors influencing adherence.

Results: Adherence to screening recommendations was significantly greater among non-Indigenous Australians (biennial screening; 77.5\%) than Indigenous Australians (annual screening; 52.7\%; $P<0.001$ ). Greater adherence by nonIndigenous Australians was associated with longer duration of diabetes (adjusted odds ratio [aOR], 1.19 per 5 years; $P=0.018$ ), while increasing age was associated with poorer adherence in non-Indigenous Australians (aOR, 0.70 per decade; $P=0.011$ ). For Indigenous Australians, residing in inner regional areas (aOR, $1.66 ; P=0.007)$ and being male (aOR, $1.46 ; P=0.018)$ were significant factors positively associated with adherence.

Conclusions: More than three-quarters of non-Indigenous Australians with diabetes and more than half of Indigenous Australians with diabetes adhere to the NHMRC eye examination guidelines. The discrepancy between the adherence rates may point to gaps in the provision or uptake of screening services in Indigenous communities, or a lack of awareness of the guidelines. A carefully integrated diabetic retinopathy screening service is needed, particularly in remote areas, to improve adherence rates.

The study also sought to determine the proportion of Australians with self-reported diabetes who adhere to the NHMRC retinal screening guidelines. We report in this article the rates of adherence to the guidelines by Indigenous (aged 40 years or more) and nonIndigenous Australians (aged 50 years or more).

\section{Methods}

\section{Study design}

Multistage, random cluster sampling was used to select 30 geographic sites in the five mainland Australian states and the Northern Territory, based on data from the 2011 Australian census 
by the Australian Bureau of Statistics. ${ }^{10}$ In the first sampling phase, Level 2 Statistical Areas (SA2s) were stratified by remoteness into major city, inner regional, outer regional, remote and very remote areas; respectively twelve, six, six, four and two SA2s were randomly selected from these categories. A Level 1 Statistical Area (SA1) or cluster of SA1s containing about 100 non-Indigenous Australians aged 50 years or more and 50 Indigenous Australians aged 40 years or more was selected from within each SA2 and designated as the recruitment site. The lower age criterion for Indigenous Australians was selected because of the younger age of onset and more rapid progression of eye disease and diabetes in this group. Recruiters proceeded door to door to recruit 1738 Indigenous Australians and 3098 non-Indigenous Australians between March 2015 and April 2016. A positive response rate of $82.5 \%$ (3729 of 4520) and a clinical examination rate of $68.5 \%$ (3098 of 4520 ) were achieved for non-Indigenous recruitment, while positive response and examination rates for Indigenous participants were 90.9\% (2035 of 2240 ) and $77.6 \%$ (1738 of 2240) respectively. Participants were examined with a series of standard clinical eye tests; the results of these examinations are not reported in this article.

\section{Interviewer-administered general questionnaire}

Information about ethnic background (including Indigenous status and country of birth), highest educational level, and history of ocular problems, stroke and diabetes was collected by an interviewer administering a questionnaire. Participants were asked whether they had been told by a doctor or nurse that they had diabetes (ie, diabetes in this study was self-reported). The age at diagnosis for those with self-reported diabetes was recorded. Participants were asked whether they had seen an ophthalmologist or optometrist for a diabetic eye examination, and if so, how long ago (in years). This information was used to determine the proportion of participants who adhered to the NHMRC guidelines. Those who reported that they had not undergone a diabetic eye check were asked for a reason, and their answer was recorded according to a standardised list: "I did not know about the guideline"; "I missed the appointment"; "I have no time"; and other.

\section{Statistical analysis}

The primary outcome was adherence to the NHMRC diabetic eye examination guidelines. Demographic characteristics were summarised as means and standard deviations (SDs) for normally distributed continuous data, and as medians with interquartile ranges (IQRs) for skewed data. Normality was assessed in boxplots, and with Kolmogorov-Smirnov and Shapiro-Wilks tests. Univariate and multivariable logistic regression analysis was used to identify risk factors associated with adherence. Adjusted proportions were calculated by generalised logit regression models taking into account the sampling weight and non-response rate. A plot of the residuals against estimates was examined to determine whether the assumptions of linearity and homoscedasticity were met. The Stata module NLCHECK was used to test assumptions of linearity after model estimation. A Box-Tidwell model was used to transform a predictor using power transformations to find the best power for model fit based on maximal likelihood estimate. Because of the small numbers of participants with self-reported diabetes from very remote sites (17 Indigenous and 18 nonIndigenous people), the remote and very remote strata were collapsed in the regression analysis. Variables found to be nonsignificant in univariate analysis were excluded from the multivariable model. All analyses were performed by incorporating sampling weights and non-response rate. Analyses were conducted in Stata 14.2.0 (StataCorp). $P<0.05$ (two-tailed) was deemed statistically significant.

\section{Ethics approval}

The protocol for this study was approved by the Royal Victorian Eye and Ear Hospital Human Research Ethics Committee (reference, HREC-14/1199H). Additional ethics approvals for conducting research in Indigenous communities were obtained at the state level. The research complied with the tenets of the Declaration of Helsinki.

\section{Results}

\section{Study participants}

A total of 1738 Indigenous Australians (men, 41.1\%) aged 40-92 years (mean, 55.0 years; SD, 10.0 years) were examined, of whom 645 (men, 37.5\%; mean age, 68.6 years [SD, 8.9]) had self-reported diabetes (crude prevalence, $37.1 \%$; age- and sampling-adjusted prevalence, $42.6 \%$ ). Of the 3098 non-Indigenous Australians (men, 46.4\%) aged 50-98 years (mean, 66.6 years; SD, 9.7 years) examined, 431 had self-reported diabetes (men, 58.3\%; mean age, 58.3 years [SD, 9.8]); the prevalence was significantly lower than for the Indigenous participants (crude prevalence, 13.9\%; adjusted prevalence, $13.3 \% ; P<0.001$ ). The mean age of non-Indigenous participants who adhered to the NHMRC diabetic eye examination guidelines was 68.4 years (SD, 8.7 years), and the median duration of diabetes since diagnosis was 10 years (IQR, 5-18 years). The mean age of Indigenous Australians who had undergone recommended eye examinations was 58.7 years (SD, 9.7 years), and the median duration of diabetes was 11 years (IQR, 5-20 years) (Box 1 ).

\section{Adherence to the NHMRC diabetic eye examination guidelines}

More than half the Indigenous participants with self-reported diabetes (341 participants; unadjusted rate, 52.9\%; age- and sampling-adjusted rate, $52.7 \%$ ) reported that they had had a diabetic eye examination in the past 12 months, in accordance with the NHMRC guidelines. Of the non-Indigenous Australians with selfreported diabetes, $77.7 \%$ (adjusted rate, 77.5\%) had had eye examinations in the preceding 2 years ( $v$ Indigenous proportion: $P<0.001$; Box 2). In total, $26.2 \%$ of Indigenous Australians with diabetes reported that they had never undergone a diabetic eye examination, compared with $15.3 \%$ of non-Indigenous participants $(P<0.001)$.

The major reason for non-adherence reported by both Indigenous $(72.6 \%)$ and non-Indigenous participants $(74.3 \%)$ was that they were unaware of the need for regular eye examinations.

\section{Effects of associated risk factors on adherence to the NHMRC guidelines}

Indigenous and non-Indigenous Australians combined. Univariate logistic regression indicated that Indigenous status was significantly associated with a lower likelihood of having adhered to the NHMRC guidelines (odds ratio [OR], 0.37; $P<0.001$ ). After adjusting for covariates, longer duration of diabetes (OR, 1.11 per 5 years; $P=0.012$ ) and inner regional residence (adjusted OR [aOR], 1.60; $P=0.012$ ) were associated with a greater likelihood of adhering, while Indigenous status remained a strong risk factor for non-adherence (aOR, 0.29; $P<0.001)$ (Box 3).

Indigenous and non-Indigenous Australians separately. Data for Indigenous and non-Indigenous participants were also analysed separately to account for differences in inclusion criteria. In non-Indigenous Australians, univariate and multivariate 
1 Socio-demographic characteristics of participants with self-reported diabetes who adhered or did not adhere to National Health and Medical Research Council guidelines for retinopathy screening (unadjusted for age or sampling)

\begin{tabular}{|c|c|c|c|c|}
\hline & \multicolumn{2}{|c|}{ Non-Indigenous } & \multicolumn{2}{|c|}{ Indigenous } \\
\hline & Adherers & Non-adherers & Adherers & Non-adherers \\
\hline Number of participants & 335 & 96 & 341 & 304 \\
\hline Age (years), mean (SD) & $68.4(8.7)$ & $69.6(9.7)$ & $58.7(9.7)$ & $57.8(9.8)$ \\
\hline Duration of diabetes (years), median (IQR) & $10.0(5-18)$ & $8.0(3-14)$ & $11.0(5-20)$ & $10.0(5-19)$ \\
\hline Education (years), mean (SD) & $12.2(3.9)$ & $11.7(3.4)$ & $10.7(3.9)$ & $10.3(3.2)$ \\
\hline Sex (male) & $181(54.0 \%)$ & $61(64 \%)$ & $131(38.4 \%)$ & 111 (36.5\%) \\
\hline English spoken at home & $298(89.0 \%)$ & $85(88 \%)$ & $318(93.3 \%)$ & $288(94.7 \%)$ \\
\hline \multicolumn{5}{|l|}{ Place of birth } \\
\hline Oceania & $231(69 \%)$ & $56(58 \%)$ & $340(99.7 \%)$ & $303(99.7 \%)$ \\
\hline Europe & $70(21 \%)$ & $27(28 \%)$ & $1(0.3 \%)$ & $1(0.3 \%)$ \\
\hline Other & $34(10 \%)$ & $13(14 \%)$ & 0 & 0 \\
\hline
\end{tabular}

analysis showed that older age was a risk factor for non-adherence (aOR, 0.70 per decade; $P=0.011$ ), while longer duration of diabetes was associated with greater likelihood of adhering (aOR, 1.19 per 5 years; $P=0.018)$. Among Indigenous participants, adherence was greater for men $(\mathrm{aOR}, 1.46 ; P=0.018)$ and for those in inner regional areas (aOR, 1.66; $P=0.007)$ (Box 3$)$.

\section{Discussion}

The adjusted prevalence of self-reported diabetes among participants in the NEHS was more than three times higher for Indigenous than for non-Indigenous participants (42.6\% v 13.3\%). Almost $80 \%$ of non-Indigenous participants and half the Indigenous participants with diabetes reported that they had adhered to the NHMRC retinopathy screening guidelines. Longer duration of diabetes was associated with greater adherence and greater age with non-adherence in the non-Indigenous group, while among Indigenous participants those who were men or living in an inner regional locality were more likely to have adhered to the recommendations.

The adherence rate of $77.5 \%$ for non-Indigenous Australians matches the $77 \%$ reported in the AusDiab study. ${ }^{12}$ However, AusDiab included participants from a much broader age range (25 years and over) and can therefore be compared with the older NEHS sample only with caution. A more relevant comparison is with the adherence

2 Rates of adherence to the National Health and Medical Research Council guidelines for retinopathy screening,* by Indigenous status and residential location

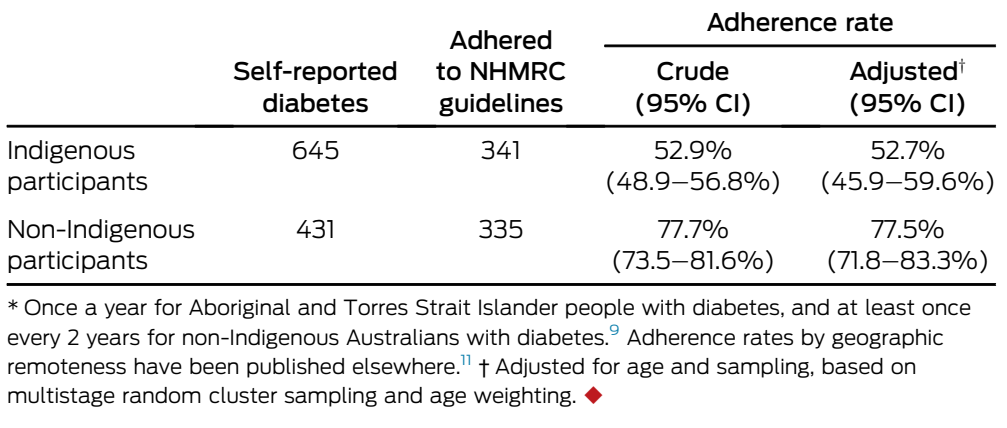

rate of about $50 \%$ in the older population of the Melbourne Visual Impairment Project. ${ }^{13}$ The higher adherence rate we found may indicate improved access to and awareness of diabetic retinopathy screening services among people with diabetes, or improved awareness of the guidelines among health care providers. ${ }^{14}$ In any case, adherence rates must improve further to compensate for the expected rise in the incidence of diabetes. ${ }^{3}$ Australia would benefit from a carefully integrated diabetic retinopathy screening system that ensures coverage for all Australians with diabetes, particularly in underserviced remote areas. Combined with improvements in referral pathways for those identified as having vision-threatening retinopathy and increased education of those with diabetes about examination guidelines, an integrated and accessible screening program would increase the uptake of eye examinations. Such programs have been effective in other countries, ${ }^{15}$ and would improve the management of diabetic retinopathy in Australia.

The proportion of Indigenous adults with diagnosed diabetes who had had an eye examination during the past 12 months has increased since the completion of the National Indigenous Eye Health Survey (NIEHS) in 2008, from about 20\% to more than $50 \%{ }^{16,17}$ This suggests that interventions in Indigenous eye health since the NIEHS may be having a significant impact. ${ }^{18}$ Despite this improvement, an unacceptably high proportion of Indigenous Australians with diabetes are not having potentially vision-saving examinations. Coinciding with these findings, the Australian government has allocated $\$ 33.8$ million in Medicare rebates to general practitioners for non-mydriatic fundus photography in diabetic patients. ${ }^{19}$ Primary health care providers are at the frontline of diabetes care, and integrating regular fundus photography by GPs into their routine management of diabetes should significantly reduce the burden of diabetes-related vision loss. The new Medicare rebate will be of particular benefit to under-resourced Indigenous health services in the most remote regions of Australia. Indigenous Australians in very remote communities are at particularly high risk of diabetic retinopathy, and the lowest adherence rate in the NEHS - 38\% in very remote areas - indicates that integrating regular retinal screening into primary care in these communities is essential for improving Indigenous eye health. ${ }^{17}$

The positive association between regular eye examinations and disease duration has important implications 
3 Factors associated with adherence to the National Health and Medical Research Council guidelines for retinopathy screening: univariate and multivariate logistic regression*

\begin{tabular}{c} 
All participants \\
\hline OR $(95 \% \mathrm{Cl})$
\end{tabular}

\begin{tabular}{ll} 
Non-Indigenous & \\
\hline OR $(95 \% \mathrm{Cl})$ & $P$
\end{tabular}

\begin{tabular}{ll}
\multicolumn{2}{c}{ Indigenous } \\
\hline OR $(95 \% \mathrm{Cl})$ & $P$
\end{tabular}

Univariate analysis
Age (per 10 years)
Duration of diabetes (per 5 years)
Education (per year)
Sex (male)
Indigenous
English spoken at home
Place of birth
Oceania
Europe
Other

$1.12(0.93-1.36)$
$1.06(0.98-1.15)$
$1.06(0.99-1.13)$
$1.18(0.85-1.64)$
$0.37(0.25-0.56)$
$0.86(0.52-1.42)$

\subsection{4}

0.14

0.08

0.32

$<0.001$

$0.86(0.52-1.42)$

1

$1.49(0.77-2.89)$

0.23

0.94

0.54
0.021

0.018

0.53

0.09

$1.06(0.65-1.73)$

0.80

$$
\begin{gathered}
1.00(0.68-1.46) \\
1.02(0.93-1.11) \\
1.06(0.94-1.18) \\
1.37(1.02-1.85)
\end{gathered}
$$

$1.19(0.43-3.30)$

0.72

1

$\begin{array}{ll}0.74(0.41-1.34) & 0.30 \\ 0.51(0.21-1.26) & 0.14\end{array}$

Remoteness

Major city
Inner regional
Outer regional
Remote/very remote
Multivariate analysis
Age (per 10 years)
Duration of diabetes (per 5 years)
Education (per year)
Sex (male)
Indigenous
Place of birth
Oceania
Europe
Other

\section{1}

$1.64(0.94-2.88)$

$1.18(0.61-2.30)$

$1.55(0.87-2.74)$

$0.85(0.69-1.04)$

$1.11(1.02-1.20)$

$1.04(0.98-1.11)$

0.94 (0.69-1.28)

$0.29(0.18-0.47)$

1

$0.75(0.44-1.27)$

$0.43(0.13-1.39)$
0.08

0.60

0.13

0.11

0.012

0.23

0.70

$<0.001$

0.15
1

$1.68(0.89-3.16)$

1.39 (0.77-2.49)

$1.49(0.73-3.04)$

$0.70(0.54-0.92)$

1.19 (1.03-1.37)

$1.04(0.96-1.12)$

$0.61(0.36-1.11)$

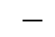

1

$0.74(0.44-1.24)$

0.24

$0.39(0.09-1.70)$

0.10

0.26

0.26

0.011

0.018

0.31

$-$
0.069

1

$1.60(1.12-2.29)$

$1.10(0.71-1.70)$

1.35 (0.83-1.29)
1

0.012

0.66

0.22
$1.53(0.78-3.02)$

$1.29(0.69-2.42)$

$1.66(0.86-3.24)$

\subsection{0}

$\mathrm{OR}=$ odds ratio. $*$ Analysis adjusted for sampling weight and non-response rate.

for treating and preventing diabetic retinopathy. The prevalence of diabetic retinopathy increases with duration of disease; $60 \%$ of people with type 2 diabetes and almost all with type 1 diabetes develop retinopathy within 20 years of diagnosis. ${ }^{20}$ Regular screening is therefore particularly important for those who have had diabetes for many years. Lower adherence by people with a shorter duration of diabetes may be noteworthy, as detecting retinopathy early is associated with better treatment outcomes. ${ }^{5}$ That increasing age was associated with a significantly lower likelihood of adherence highlights a clear need to improve the uptake of diabetes eye care services by older people. As our population is ageing, the prevalence of diabetes-related vision loss is likely to increase if older Australians with diabetes neglect their eye health. ${ }^{21}$

The primary limitation of our study was the use of self-reported data for ascertaining diabetes; we cannot exclude the possibility of self-reporting bias. In addition, recall bias may have led patients to over- or underestimate the time since their most recent eye examination. Similarly, participants may not have accurately recalled whether their fundus had been investigated during this examination. Nevertheless, self-reports are commonly used for diabetes surveillance, $^{22,23}$ and earlier studies have reported excellent sensitivities and specificities for self-reported diabetes when compared with medical diagnoses. ${ }^{24,25}$

In summary, our findings indicate that adherence rates to the NHMRC diabetic eye examination guidelines are significantly higher than previously estimated in Australia. Despite this encouraging finding, it is important that about half of all Indigenous Australians and almost one-quarter of non-Indigenous Australians with diabetes did not have their eyes examined at the recommended frequency. While the recent allocation by the federal government of funding for screening modalities in general practice 
may further improve adherence rates, implementing an accessible integrated screening service is needed to eliminate diabetic retinopathy as a significant public health problem in Australia.

Acknowledgements: The Centre for Eye Research Australia (CERA) and Vision 2020 Australia recognise the contributions of all the National Eye Health Survey project steering committee members and the core CERA research team who assisted with the survey field work. Further, we acknowledge the overwhelming support from all collaborating Indigenous organisations who assisted with implementing the survey, and the Indigenous health workers and volunteers at each survey site who contributed to the field work. The National Eye Health Survey was funded by the Australian government, and also received financial contributions from Novartis Australia and in-kind support from our industry and sector partners, OPSM, Carl Zeiss, Designs for Vision, the Royal Flying Doctor Service, Optometry Australia and the Brien Holden Vision Institute. We specifically acknowledge OPSM, who kindly donated sunglasses to each study participant. The Centre for Eye Research Australia receives operational infrastructure support from the Victorian government. The principal investigator, Mohamed Dirani, is supported by a National Health and Medical Research Council Career Development Fellowship (\#1090466). Joshua Foreman is supported by an Australian Postgraduate Award.

Competing interests: No relevant disclosures.

Received 18 Aug 2016, accepted 7 Dec 2016.

(c) 2017 AMPCo Pty Ltd. Produced with Elsevier B.V. All rights reserved.
1 NCD Risk Factor Collaboration. Worldwide trends in diabetes since 1980: a pooled analysis of 751 population-based studies with 4.4 million participants. Lancet 2016; 387: 1513-1530.

2 Pascolini D, Mariotti SP. Global estimates of visual impairment: 2010. Br J Ophthalmol 2012; 96: 614-618.

3 Magliano DJ, Peeters A, Vos T, et al. Projecting the burden of diabetes in Australia: what is the size of the matter? Aust N Z J Public Health 2009; 33: 540-543.

4 Yau JW, Rogers SL, Kawasaki R, et al. Global prevalence and major risk factors of diabetic retinopathy. Diabetes Care 2012; 35: 556-564.

5 Ferris FL. How effective are treatments for diabetic retinopathy? JAMA 1993; 269: 1290-1291.

6 Australian Diabetes Society for the Department of Health and Ageing. Guidelines for the management of diabetic retinopathy Canberra: National Health and Medical Research Council, 2008. https://www.nhmrc. gov.au/_files_nhmrc/publications/attachments/di15.pdf (accessed Mar 2017).

7 Xie J, Arnold AL, Keeffe J, et al. Prevalence of selfreported diabetes and diabetic retinopathy in indigenous Australians: the National Indigenous Eye Health Survey. Clin Exp Ophthalmol 2011; 39: 487-493.

8 Larizza MF, Hodgson LA, Fenwick EK, et al. Feasibility of screening for diabetic retinopathy at an Australian pathology collection service: a pilot study. Med J Aust 2013; 198: 97-99. https://www.mja.com.au/journal/ 2013/198/2/feasibility-screening-diabetic-retinopathyaustralian-pathology-collection

9 Magliano DJ, Shaw JE, Shortreed SM, et al. Lifetime risk and projected population prevalence of diabetes. Diabetologia 2008; 51: 2179-2186.
10 Australian Bureau of Statistics. Australian Statistical Geography Standard (ASGS) [website] Updated June 2014. http://www.abs.gov.au/ websitedbs/d3310114.nsf/home/ australian+statistical+geography+standard + (asgs) (accessed Mar 2017).

11 Foreman J, Keel S, Xie J, et al. The National Eye Health Survey 2016: full report of the first national survey to determine the prevalence and major causes of vision impairment and blindness in Australia. Melbourne: Centre for Eye Research Australia and Vision 2020 Australia, 2016. http://www.vision2020australia.org.au/ uploads/resource/250/National-Eye-Health-Survey_ Full-Report_FINAL.pdf (accessed Mar 2017).

12 Tapp RJ, Zimmet PZ, Harper CA, et al. Diabetes care in an Australian population: frequency of screening examinations for eye and foot complications of diabetes. Diabetes Care 2004; 27: 688-693.

13 McCarty CA, Lloyd-Smith CW, Lee SE, et al. Use of eye care services by people with diabetes: the Melbourne Visual Impairment Project. Br J Ophthalmol 1998; 82: 410-414.

14 Turner AW, Mulholland WJ, Taylor HR. Coordination of outreach eye services in remote Australia. Clin Exp Ophthalmol 2011; 39: 344-349.

15 Scanlon PH, Aldington SJ, Leal J, et al. Development of a cost-effectiveness model for optimisation of the screening interval in diabetic retinopathy screening. Health Technol Assess 2015; 19: 1-116.

16 Tapp RJ, Boudville Al, Abouzeid M, et al. Impact of diabetes on eye care service needs: the National Indigenous Eye Health Survey. Clin Exp Ophthalmol 2015; 43: 540-543.

17 Turner AW, Xie J, Arnold AL, et al. Eye health service access and utilization in the National Indigenous Eye Health Survey. Clin Exp Ophthalmol 2011; 39: 598-603.
18 Tapp RJ, Anjou MD, Boudville Al, Taylor HR. The roadmap to close the gap for vision-diabetes-related eye care in the Indigenous Australian population. Diabet Med 2013; 30: 1145-1146.

19 Australian Government Department of Health. Budget 2016-17: Portfolio budget statements 2016-17; Budget related paper Nol.10. Health portfolio. Canberra: 2016. https://www.health.gov.au/internet/budget/ publishing.nsf/Content/2016-2017_Health_PBS_supl/ \$File/2016-17_Health_PBS_0.0_Complete.pdf (accessed Mar 2017)

20 Kostraba JN, Klein R, Dorman JS, et al. The epidemiology of diabetes complications study. IV. Correlates of diabetic background and proliferative retinopathy. Am J Epidemiol 1991; 133: 381-391.

21 Dunstan DW, Zimmet PZ, Welborn TA, et al. The rising prevalence of diabetes and impaired glucose tolerance: the Australian Diabetes, Obesity and Lifestyle Study. Diabetes Care 2002; 25: 829-834.

22 Welborn T, Knuiman M, Bartholomew H, Whittall D. 1989-90 National Health Survey: prevalence of self-reported diabetes in Australia. Med J Aust 1995; 163 : 129-133.

23 Xie J, Arnold A, Keeffe J, et al. Prevalence of selfreported diabetes and diabetic retinopathy in Indigenous Australians: the National Indigenous Eye Health Survey. Clin Exp Ophthalmol 2011; 39: 487-493.

24 Goldman N, Lin I, Weinstein M, Lin Y. Evaluating the quality of self-reports of hypertension and diabetes. J Clin Epidemiol 2003; 56: 148-154.

25 Huerta J, Tormo M, Egea-Caparrós J, et al. Accuracy of self-reported diabetes, hypertension, and hyperlipidemia in the Spanish population. DINO study findings. Rev Esp Cardiol 2009; 62: 143-152. 